\title{
Cetuximab-mediated ADCC activity is correlated with the cell surface expression level of EGFR but not with the $K R A S / B R A F$ mutational status in colorectal cancer
}

\author{
YUKI SEO, YOSHIYUKI ISHII, HIROKI OCHIAI, KAZUMASA FUKUDA, \\ SHINGO AKIMOTO, TETSU HAYASHIDA, KOJI OKABAYASHI, MASASHI TSURUTA, \\ HIROTOSHI HASEGAWA and YUKO KITAGAWA
}

Department of Surgery, Keio University School of Medicine, Shinjuku-ku, Tokyo 160-8582, Japan

Received January 16, 2014; Accepted February 14, 2014

DOI: 10.3892/or.2014.3077

\begin{abstract}
Cetuximab, an IgG1 monoclonal antibody against the epidermal growth factor receptor (EGFR), is widely used for the treatment of metastatic colorectal cancer (mCRC). One of the mechanisms of action is considered to be antibodydependent cell-mediated cytotoxicity (ADCC) triggered by $\mathrm{Fc} \gamma-\mathrm{R}$ on natural killer cells. However, whether ADCC is associated with EGFR expression and/or the mutational status of EGF downstream effectors ( $K R A S$ and $B R A F$ ) in colorectal cancer (CRC) remains unclear. The aim of the present study was to verify whether ADCC activities are associated with the cell surface expression levels of EGFR and/or the mutational status of KRAS and BRAF. Five human CRC cell lines with different cell surface expression levels of EGFR and different $K R A S$ and $B R A F$ mutational statuses were selected to evaluate ADCC activity using peripheral blood mononuclear cells (PBMCs) from healthy human donors. Furthermore, tumor cells from resected specimens of CRC patients were used to evaluate the cell surface expression level of EGFR using immunohistochemistry and the KRAS and $B R A F$ mutational statuses using direct sequencing, while the ADCC activity was examined using PBMCs from the same CRC patients. A strong correlation was observed between the expression levels of EGFR and the ADCC activities in the cell lines (correlation coefficient: 0.949; $\mathrm{P}=0.003$ ). Of the 13 resected specimens, a high ADCC activity level was significantly observed in tumor cells with high expression levels of cell surface EGFR, when compared with that in the tumor cells with low expression levels $(\mathrm{P}=0.027)$. In both $\mathrm{CRC}$ cell lines and tumor cells from CRC patients, the ADCC activities were significantly
\end{abstract}

Correspondence to: Dr Yoshiyuki Ishii, Department of Surgery, Keio University School of Medicine, Shinanomachi 35, Shinjuku-ku, Tokyo 160-8582, Japan

E-mail: yishii@a2.keio.jp

Key words: colorectal cancer, epidermal growth factor receptor, cell surface expression, cetuximab, antibody-dependent cell-mediated cytotoxicity associated with the cell surface expression levels of EGFR [standard partial regression coefficients: $0.911(\mathrm{P}=0.017)$ and $0.660(\mathrm{P}=0.018)$, respectively], but not with the mutational status of $K R A S$ and $B R A F$ [standard partial regression coefficient: $-0.101(\mathrm{P}=0.631)$ and $0.160(\mathrm{P}=0.510)$, respectively]. Cetuximab-mediated ADCC activity may be correlated with the cell surface expression level of EGFR, regardless of the mutational statuses of $K R A S$ and $B R A F$, in CRC.

\section{Introduction}

The World Health Organization has reported that colorectal cancer (CRC) is the third most common cancer worldwide, accounting for 940,000 million new cases annually and nearly 500,000 deaths each year. On the other hand, the treatment outcome of CRC patients has recently improved. Patients with previously untreated metastatic colorectal cancer (mCRC) have demonstrated substantial improvements, with a median overall survival time now reaching more than 24 months (1). One of the factors responsible for this improved outcome may be the development of systemic chemotherapy, including molecular-targeted therapy, for mCRC.

Cetuximab is a human-mouse chimeric immunoglobulin G1 (IgG1) monoclonal antibody for the epidermal growth factor receptor (EGFR) that has been approved for use in patients with mCRC expressing EGFR. Some clinical studies examining cetuximab treatment in patients with $\mathrm{mCRC}$ have failed to show a significant correlation between EGFR expression and the response of patients to cetuximab therapy (2). The absence of mutations in KRAS, which is one of the downstream effectors of the EGFR signaling pathway, appears to be a reliable marker for predicting the efficacy of cetuximab therapy. However, some patients with KRAS mutations were recently reported to benefit from cetuximab therapy (3).

The proposed working mechanism of cetuximab is thought to include antibody-dependent cell-mediated cytotoxicity (ADCC) triggered by $\mathrm{Fc}$ receptors $(\mathrm{Fc} \gamma-\mathrm{R})$ on natural killer cells, macrophages and polymorphonuclear leukocytes. ADCC is a well-recognized immune effector mechanism responsible for the effect of IgG1. Previous reports have demonstrated the occurrence of ADCC in CRC cell lines in vitro $(2,4)$. However, 
whether ADCC is correlated with the cell surface expression of EGFR and/or the mutational status of downstream effectors, such as KRAS and $B R A F$ in CRC remains unclear.

We demonstrated cetuximab-mediated ADCC in human CRC cell lines and investigated whether the ADCC activities were correlated with the cell surface expression levels of EGFR and/or the mutational status of $K R A S$ and $B R A F$. Furthermore, we evaluated cetuximab-mediated ADCC activity using tumor cells from resected specimens and peripheral blood samples from the same CRC patients; we then investigated the associations between the ADCC activities and the cell surface expression levels of EGFR as well as the mutational statuses of $K R A S$ and $B R A F$, in addition to performing an assay using human CRC cell lines.

\section{Materials and methods}

Cell lines and cell culture. Nine human CRC cell lines (HT29, HCT15, HCT116, DLD-1, SW480, SW867, WiDr, CaCo-2 and LoVo) and an epidermoid carcinoma cell line (A431) with different KRAS and BRAF mutational statuses were used in the present study. HT29, SW480, CaCo-2 and A431 cells were cultured in Dulbecco's modified Eagle's medium (DMEM) supplemented with heat-inactivated $10 \%$ fetal bovine serum (FBS), $50 \mathrm{U} / \mathrm{ml}$ of penicillin/streptomycin and $4.0 \mathrm{mmol} / 1$ of glutamine. The other cell lines were maintained in complete RPMI-1640 medium with the addition of $10 \%$ heat-inactivated FBS, $100 \mathrm{U} / \mathrm{ml}$ of penicillin/streptomycin, and $2.0 \mathrm{mmol} / \mathrm{l}$ of glutamine and cultured at $37^{\circ} \mathrm{C}$ in a $5 \% \mathrm{CO}_{2}$-humidified atmosphere. Adherent cells were removed using trypsin-EDTA solution $[0.05 \%$ trypsin and $0.02 \%$ EDTA in phosphatebuffered saline (PBS) without calcium and magnesium].

ADCC assay using human CRC cell lines. Cetuximabmediated ADCC activity was evaluated using a 24-h lactate dehydrogenase (LDH)-releasing assay, the CytoTox 96 nonradioactive cytotoxicity assay kit (Promega, Madison, WI, USA), according to the manufacturer's protocol.

Target tumor cells (HT29, HCT116, DLD-1, SW480, CaCo-2 and A431) with different $K R A S$ and $B R A F$ mutational statuses (Table I) (5-10) were seeded at a concentration of $1 \times 10^{5}$ cells $/ \mathrm{ml}$ in a 96-well plate. After $24 \mathrm{~h}$, the cells were exposed to cetuximab or non-specific mouse and human IgG as a control at concentrations of 0,10 and $100 \mu \mathrm{g} / \mathrm{ml}$ in a $5 \% \mathrm{CO}_{2}$ incubator for $1 \mathrm{~h}$. Then, the cells were cultured with peripheral blood mononuclear cells (PBMCs) in a $5 \% \mathrm{CO}_{2}$ incubator for $4 \mathrm{~h}$. The PBMCs were freshly prepared from healthy human donors, isolated from heparinized peripheral blood using a Ficoll gradient, and added to wells at each effector:target (E:T) cell concentration (E:T ratios of 20:1 and 10:1).

Cell lysis was determined by measuring the amount of released LDH in the culture supernatants. ADCC was evaluated using the following formula: \% Cytotoxicity $=[$ (experimental - effector spontaneous control - target spontaneous control)/ (target maximum release - target spontaneous control)] x 100 .

Flow cytometric analysis. The cell surface expression of EGFR in the CRC cell lines was quantified using a flow cytometric system (FACSVantage SE; Becton-Dickinson, San Jose, CA, USA). The binding of cetuximab to the CRC cell lines
Table I. Cell surface expression levels of EGFR and mutational status of downstream effectors in the cell lines.

\begin{tabular}{lrllc}
\hline $\begin{array}{l}\text { Cell } \\
\text { lines }\end{array}$ & $\begin{array}{c}\text { EGFR } \\
(\%)\end{array}$ & \multicolumn{1}{c}{ KRAS } & BRAF & $\begin{array}{c}\text { ADCC } \\
\text { activity (\%) }\end{array}$ \\
\hline A431 & 99.8 & Wild & Mutant & 67 \\
CaCo-2 & 89.6 & Wild & Wild & 70 \\
SW480 & 88.8 & Mutant (G12V) & Wild & 59 \\
DLD-1 & 53.4 & Mutant (G13D) & Wild & 61 \\
HT29 & 6.9 & Wild & Mutant & 26 \\
HCT116 & 0.1 & Mutant (G13D) & Wild & 19 \\
\hline
\end{tabular}

The mutational statuses of $K R A S$ and $B R A F$ was identified in previous reports (5-10).

was titrated using a flow cytometric analysis. Biotinylated cetuximab (11) and another anti-EGFR antibody (PE mouse anti-human EGF receptor; BD Biosciences, San Jose, CA, USA) were used as primary antibodies. For the analysis using biotinylated cetuximab, biotin was conjugated to cetuximab using an adaptation of the method described by Medical \& Biological Laboratories Co., Ltd. (Nagoya, Japan). Then, 1x10 tumor cells were incubated with $1 \mu \mathrm{g} / \mathrm{ml}$ of biotinylated cetuximab in $1 \%$ bovine serum albumin in PBS for $1 \mathrm{~h}$ at room temperature. The cell surface was stained with streptavidin for $15 \mathrm{~min}$ at room temperature in the dark. For the analysis using PE mouse anti-human EGFR, $1 \mu \mathrm{g}$ of anti-EGFR antibody per $1 \times 10^{6}$ tumor cells was used as the primary antibody. Ten micrograms per milliliter of fluorescent-labeled anti-mouse IgG was used as the secondary antibody. The samples were then washed three additional times with cold PBS, resuspended in $500 \mu \mathrm{l}$ of PBS, and analyzed using flow cytometry. For each sample, $2 \times 10^{4}$ events were acquired. The analysis was performed by the triplicate determination of at least three separate experiments. The expression levels were described as the percentage of positive cells (number of positive-stained cells x 100/total number of cells).

Ex vivo ADCC assay using tumor cells of resected specimens and PBMCs from CRC patients. Cetuximab-mediated ADCC was also examined using tumor cells and PBMCs isolated from the same CRC patients $(\mathrm{n}=13)$ with pathological stage II or III disease (American Joint Committee on Cancer). A portion of the resected CRC specimens was obtained from the CRC patient as soon as possible when bowel resection was performed at our institution. The specimens were cut using scissors to form single cells and were incubated at a concentration of $1 \times 10^{5}$ cells $/ \mathrm{ml}$ in a 96-well plate overnight. At the time of the experiment, PBMCs were freshly separated from the same CRC patient. The cells were then exposed to cetuximab or non-specific mouse and human IgG as a control at concentrations of 0,10 and $100 \mu \mathrm{g} / \mathrm{ml}$ for $1 \mathrm{~h}$, and the PBMCs were subsequently added to the wells at each E:T cell concentration (E:T ratios of 20:1 and 10:1). The subsequent protocol was the same as that used for the in vitro ADCC assay described above. 
ONCOLOGY REPORTS 31: 2115-2122, 2014

2117

A

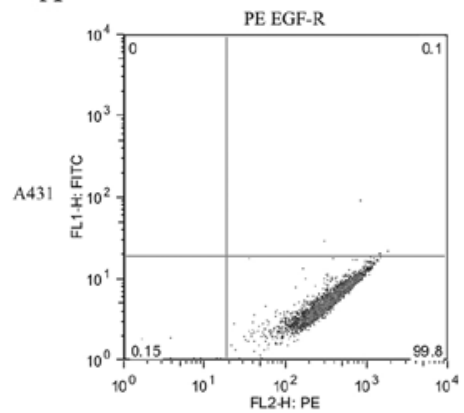

B

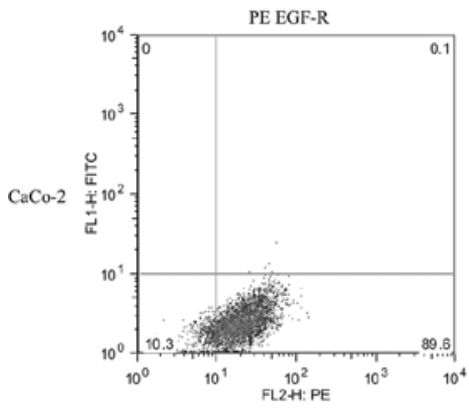

C

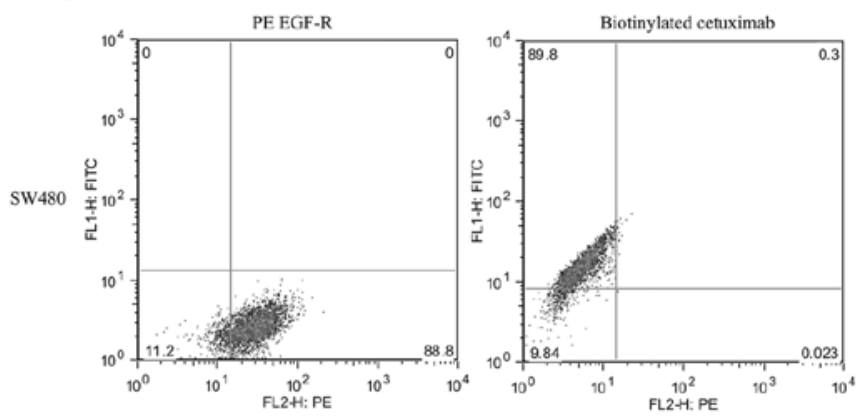

D
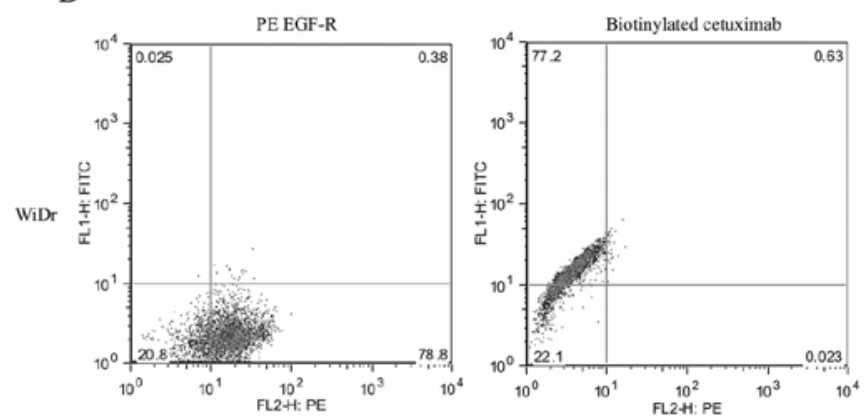

E
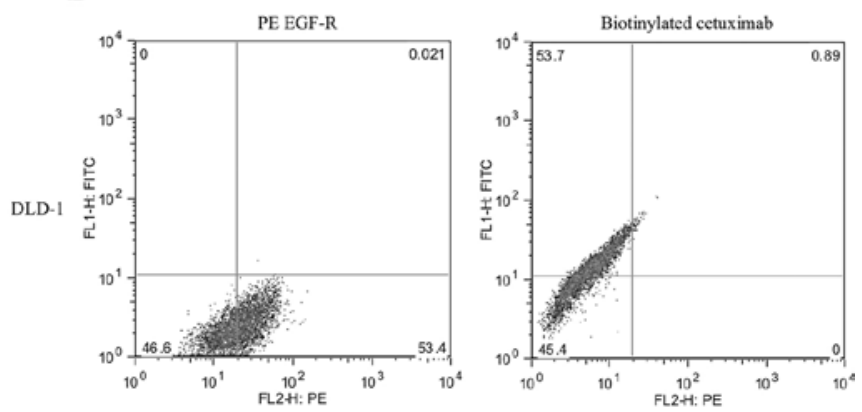

$\mathrm{F}$

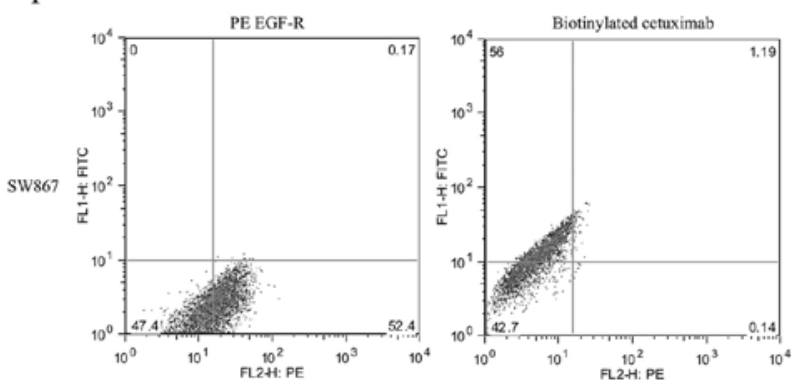

G

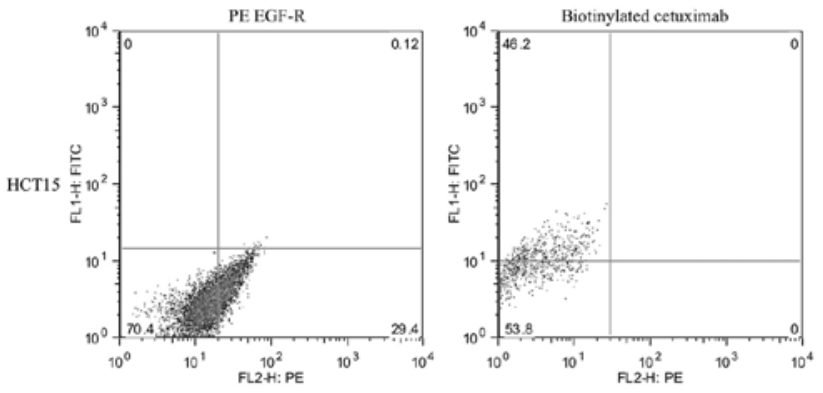

$\mathrm{H}$
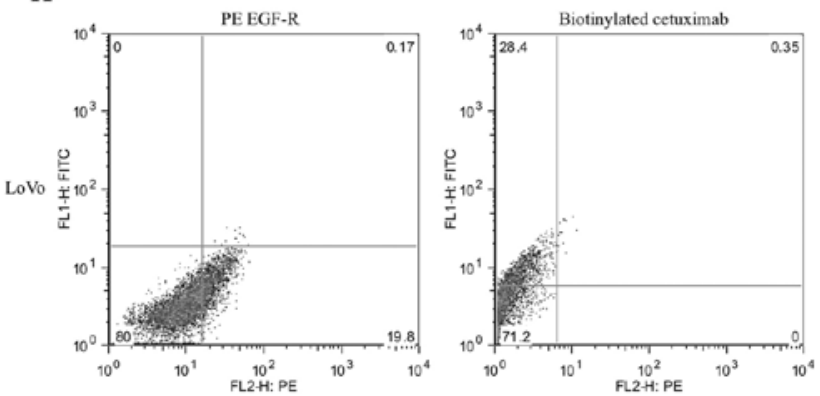

I
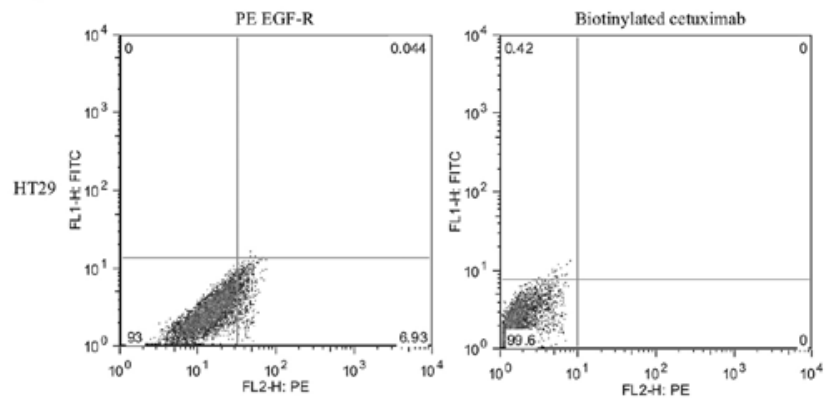

$\mathrm{J}$
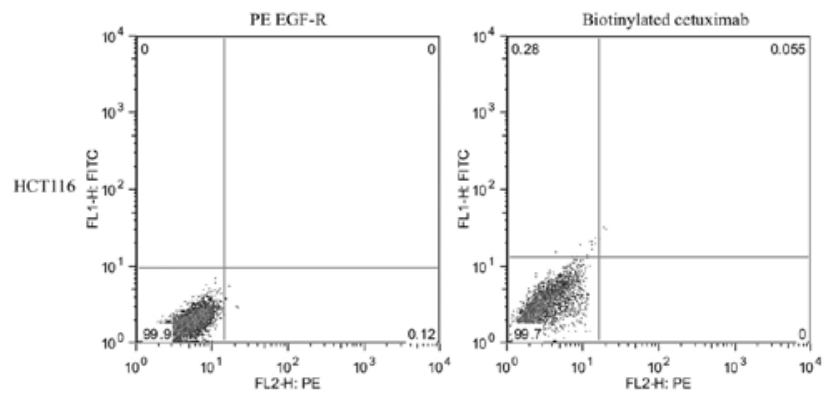

Figure 1. Cell surface expression of EGFR as determined by flow cytometric analysis in colorectal cancer cell lines: (B) CaCo-2, (C) SW480, (D) WiDr, (E) DLD-1, (F) SW867, (G) HCT15, (H) LoVo, (I) HT29, (J) HCT116, and an epidermoid carcinoma cell line (A) A431 as a positive control. The primary antibodies were PE mouse anti-human EGFR and biotinylated cetuximab. 
Immunohistochemistry. The CRC specimens were immersed in $4 \%$ buffered neutral formalin and fixed for $24 \mathrm{~h}$. Paraffinembedding was performed according to standard procedures. Sections $(4 \mu \mathrm{m})$ were mounted on coated slides and allowed to dry for $30 \mathrm{~min}$ at $60^{\circ} \mathrm{C}$ and overnight at $37^{\circ} \mathrm{C}$. All of the sections were stained in Estisol 220 (Esti Chem, Køge, Denmark) and rehydrated in graded alcohol solutions. Endogenous peroxidase was blocked with $3 \%$ hydrogen peroxide. Proteolytic antigen retrieval was performed using $0.1 \%$ protease at room temperature for $20 \mathrm{~min}$. The slides were incubated in the primary anti-human EGFR antibody (clone DAK-H1-WT; Dako, Copenhagen, Denmark) for $30 \mathrm{~min}$ at room temperature. Visualization of the reaction was performed using EnVision + DAB (Dako Cytomation-DK) followed by counterstaining with hematoxylin. All the staining procedures were manually performed at one time.

Evaluation of immunohistochemical variables. The expression of EGFR was quantified using a visual grading system based on the American Society of Clinical Oncology/College of American Pathologists guidelines for human epidermal growth factor receptor 2 (HER-2) (12), and the intensity of membranous staining was graded on a scale of 0 to $3+(0$, percentage of positive tumor cells $<10 \%, 1+$, percentage of positive tumor cells $\geq 10 \%$ and weak staining; $2+$, percentage of positive tumor cells $\geq 10 \%$ and moderate staining, $3+$ : percentage of positive tumor cells $\geq 10 \%$ and strong staining). This evaluation was performed by two professional pathologists.

DNA extraction and mutation analysis. First, the tumor cells in each tumor block were histologically evaluated using hematoxylin and eosin staining. Then, DNA was extracted from formalin-fixed and paraffin-embedded samples after macrodissection. The presence of $K R A S$ and $B R A F$ was determined using an allelic discrimination assay performed on a 7500HT real-time PCR system. The samples were screened for KRAS mutations located within codons 12 and 13 and $B R A F$ mutations located within codon 600 . All of the mutations were confirmed by direct sequencing (13).

The present study was performed in accordance with the guidelines of the Declaration of Helsinki, as amended in Edinburgh, Scotland, in October 2000. The study was approved by the Institutional Review Board of Keio University Hospital. Written informed consent was obtained from each patient prior to the experiments.

Statistical considerations. The correlation between ADCC activity and the cell surface expression level of EGFR in vitro was evaluated using a univariate regression analysis. The associations between the ADCC activities and the cell surface expression levels of EGFR and/or the KRAS/BRAF mutational status in CRC were analyzed using a one-factor ANOVA or multiple regression model. P-values $<0.05$ were considered to indicate statistically significant results.

\section{Results}

Cell surface expression levels of EGFR in human CRC cell lines. The cell surface expression levels of EGFR in the human CRC cell lines were evaluated using flow cytometric analysis

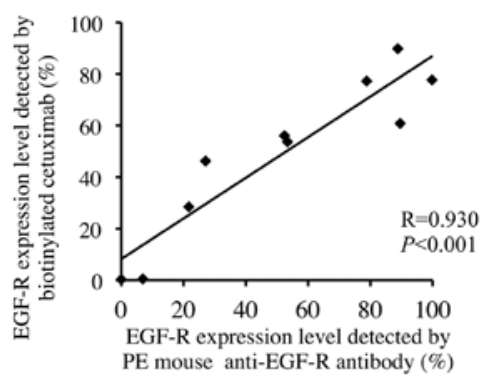

Figure 2. Correlation between the expression levels detected using biotinylated cetuximab and those detected using PE mouse anti-human EGFR antibody in each of the cell lines $(\mathrm{R}=0.930, \mathrm{P}<0.001)$.

(Fig. 1). Using PE mouse anti-human EGFR as a primary antibody, the cell surface EGFR expression levels in the CaCo-2, SW480, WiDr, DLD-1 and SW867 cells were as high as that of the positive control (A431), whereas the levels in the HT29 and HCT116 cells were relatively low. The EGFR expression level in each cell line, as detected using biotinylated cetuximab, was similar to that for the PE mouse anti-human EGFR, and a strong correlation was observed between these levels, with a correlation coefficient of 0.930 ( $\mathrm{P}<0.001$; Fig. 2).

ADCC activities in human CRC cell lines. Cetuximabmediated ADCC activities were detected at various degrees in all of the CRC cell lines, and the highest ADCC activity in each cell line was detected at a cetuximab concentration of $100 \mu \mathrm{g} / \mathrm{ml}$ and an E:T ratio of 20:1 (Fig. 3). Under this condition, a strong correlation was observed between the cell surface expression levels of the EGFR and ADCC activities in the human CRC cell lines (correlation coefficient, 0.949; $\mathrm{P}=0.003$; Fig. 4). Furthermore, a multiple regression analysis using two variables (cell surface expression level of EGFR and $K R A S$ or $B R A F$ mutation status) revealed that ADCC activity was significantly associated with the cell surface expression level of EGFR (standard partial regression coefficient, 0.911; $\mathrm{P}=0.017)$, but not with the KRAS/BRAF mutational status (standard partial regression coefficient, $-0.101 ; \mathrm{P}=0.631$ ).

Cell surface expression levels of EGFR in resected tumors of CRC patients. Immunohistochemical staining for EGFR in the resected tumors is shown in Fig. 5. Of the 13 patients, a staining score of $3+$ was observed in 3 patients (cases A, B and C), a score of $2+$ was observed in 3 patients (cases D, E and F), a score of $1+$ was observed in 3 patients (cases $\mathrm{G}, \mathrm{H}$ and I), and no staining was observed in 4 patients (cases J, K, $\mathrm{L}$ and $\mathrm{M}$ ). In all cases, the EGFR staining was localized in the cell membrane.

ADCC activities in tumor cells from the CRC patients. Various degrees of cetuximab-mediated ADCC activities were also detected in the tumor cells from the CRC patients, and the highest ADCC activity in each patient was also detected at a cetuximab concentration of $100 \mu \mathrm{g} / \mathrm{ml}$ and an E:T ratio of 20:1. The profiles, including the percentage of cetuximabmediated ADCC activities under this condition and the cell surface expression levels of EGFR as determined using immunohistochemistry, for each of the patients are shown in 
A

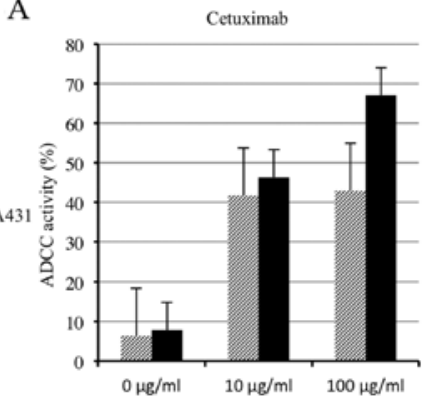

B

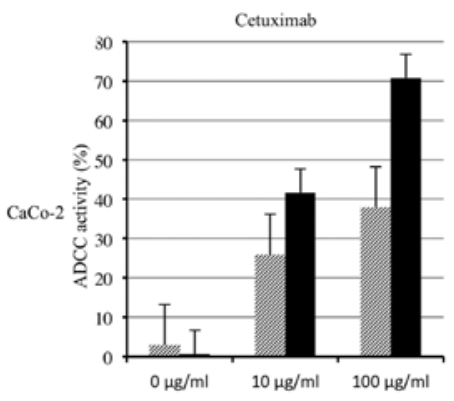

C

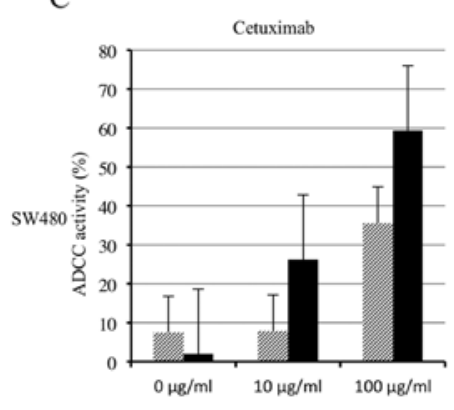

Non-specific mouse IgG

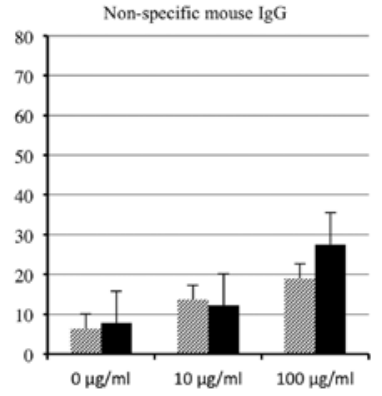

Non-specific mouse $\lg \mathrm{G}$

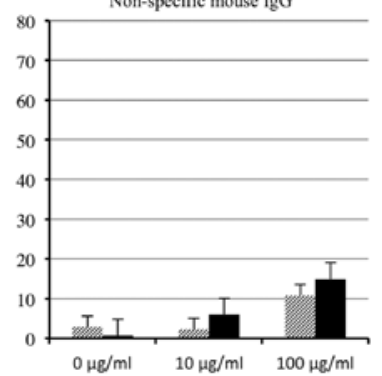

Non-specific mouse $\operatorname{lgG}$

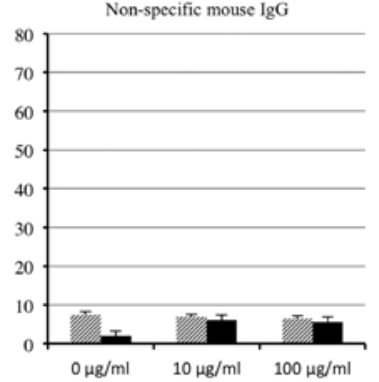

D

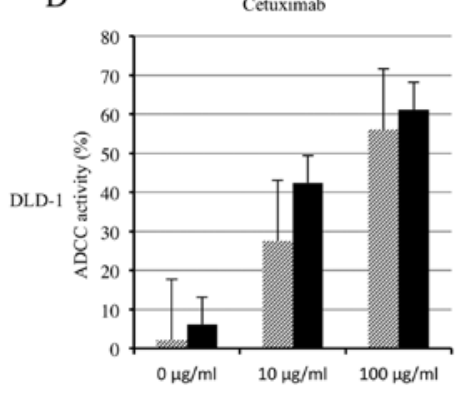

E

Cetuximab

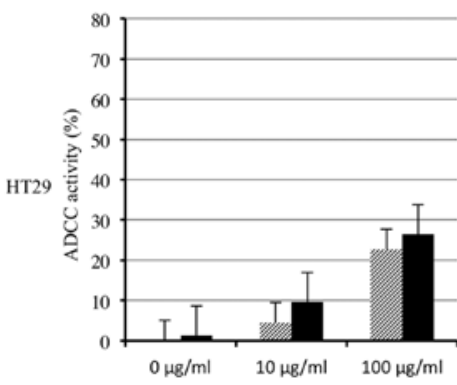

F

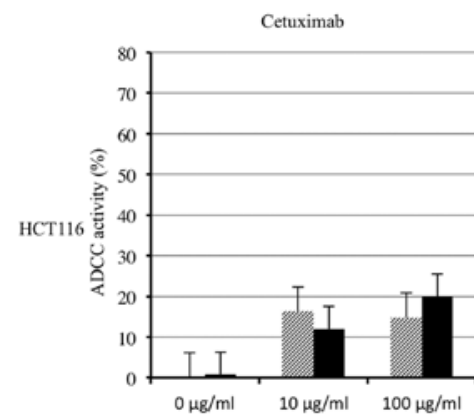

Non-specific mouse $\lg G$

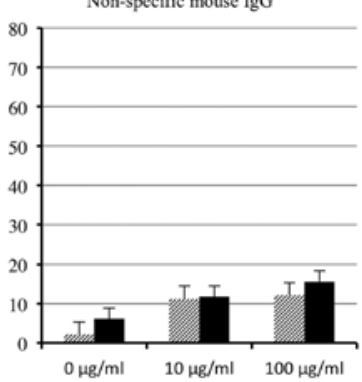

Non-specific mouse IgG

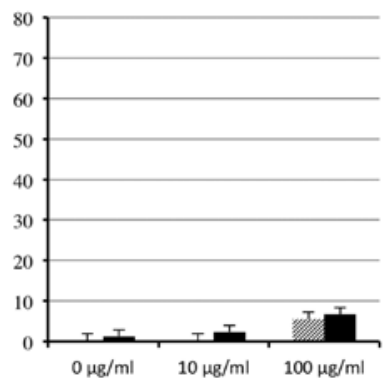

Non-specific mouse IgG

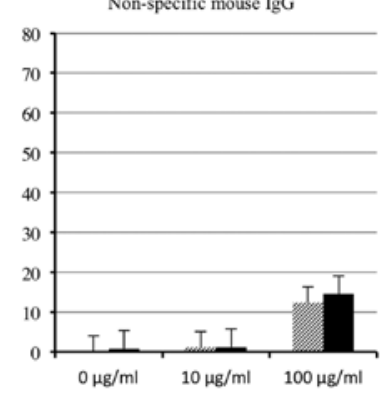

Figure 3. ADCC activities in colorectal cancer cell lines. Non-specific human IgG was used as a negative control. The highest ADCC activity in each cell line was observed under the conditions of a cetuximab concentration of $100 \mu \mathrm{g} / \mathrm{ml}$ and an E:T ratio of 20:1. (A) A431, (B) CaCo-2, (C) SW480, (D) DLD-1, (E) HT29 and (F) HCT116 cell lines. Slashed bar, E:T ratio of 10:1; black bar, E:T ratio of 20:1.

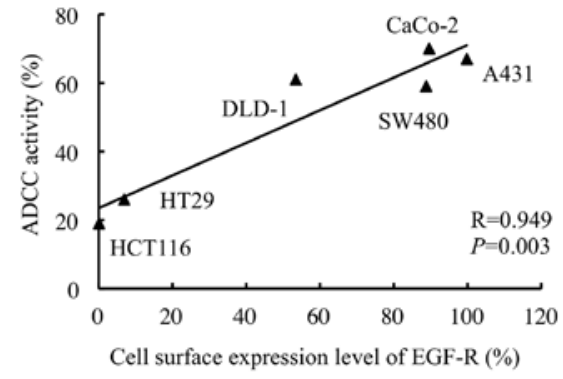

Figure 4. Correlation between cell surface expression levels of EGFR and ADCC activities in 6 cell lines, including A431 cells (correlation coefficient, $\mathrm{R}=0.930, \mathrm{P}<0.001)$.

Table II. The cetuximab-mediated ADCC activities for the tumor cells were higher in CRC patients with a high expression level of cell surface EGFR, when compared with patients with a low expression level $(\mathrm{P}=0.027$; Fig. 6). Furthermore, the ADCC activity level was significantly associated with the cell surface expression level of EGFR (standard partial regression coefficient: $0.660, \mathrm{P}=0.018$ ), but not with the $K R A S / B R A F$ mutational status (standard partial regression coefficient, $0.160, \mathrm{P}=0.510)$.

\section{Discussion}

At present, antibodies against EGFR, such as cetuximab and panitumumab, are widely used to treat CRC patients. One of the proposed mechanisms of action of antibodies against EGFR is the direct antagonization of the EGF-stimulating activation of EGFR. These antibodies block the binding of ligands, inhibit EGFR phosphorylation, induce the internalization of EGFR, and downregulate the cell surface expression of EGFR. The $K R A S$ mutational status is well known to be a predictor of the efficacy of these antibodies, although some CRC patients are unable to benefit from treatment with anti-EGFR antibodies even when KRAS mutations are not present. In fact, the mutations of several downstream effectors of EGFR signaling, such as BRAF, PTEN and PIK3CA, have been reported in CRC, and the power balance of the mutational status of these numerous downstream effectors, including KRAS, is considered to be important for the efficacy of antibodies against EGFR. 

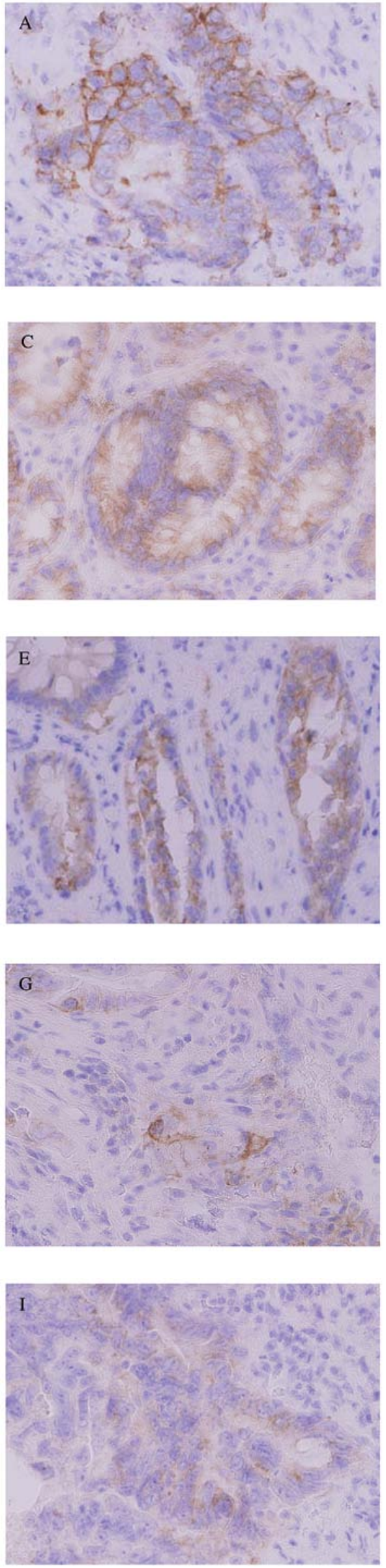

Figure 5. Immunohistochemical staining for EGFR in resected tumors from 13 colorectal cancer patients (cases A-I).
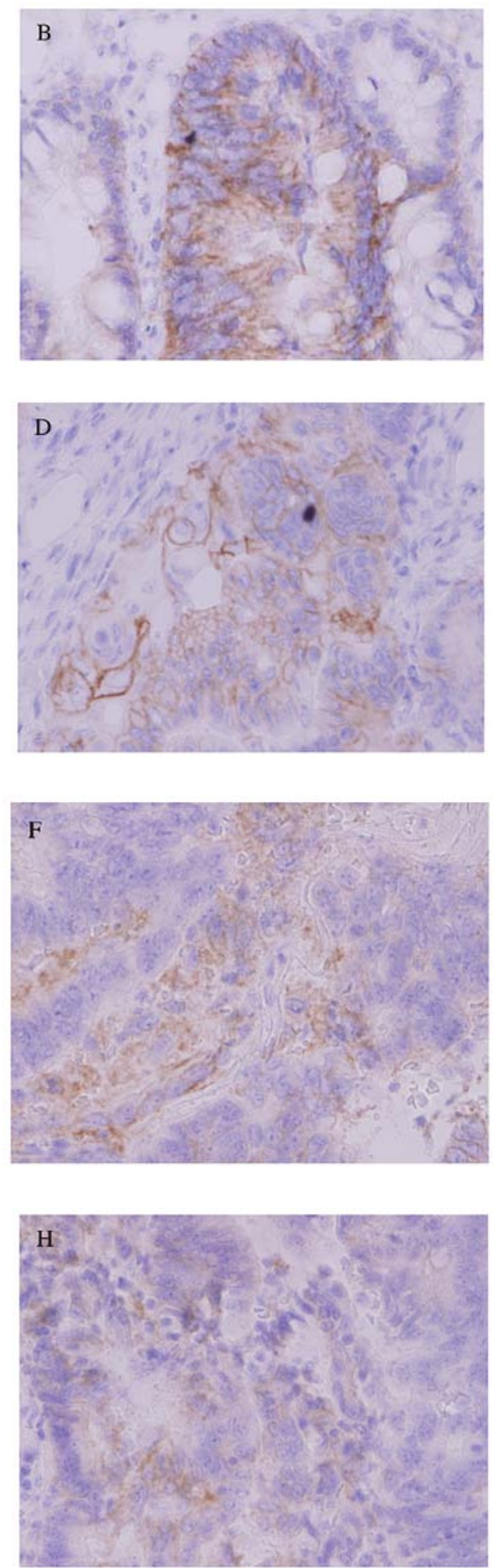

In contrast, the role of ADCC in the antitumor activity of cetuximab, which is a chimeric human-mouse IgG1 monoclonal antibody against EGFR, has not been fully investigated in CRC. Although chimeric IgG1 antibodies have been reported to induce ADCC activity in human effector cells in an efficient manner (14), the contribution of cetuximab-mediated ADCC activity to the treatment of CRC patients remains to be elucidated, and relatively little information presently exists on this topic, compared with the wide range of knowledge regarding its role in the inhibition of EGFR signaling. Therefore, the present study was designed to verify the associations between the cetuximab- 

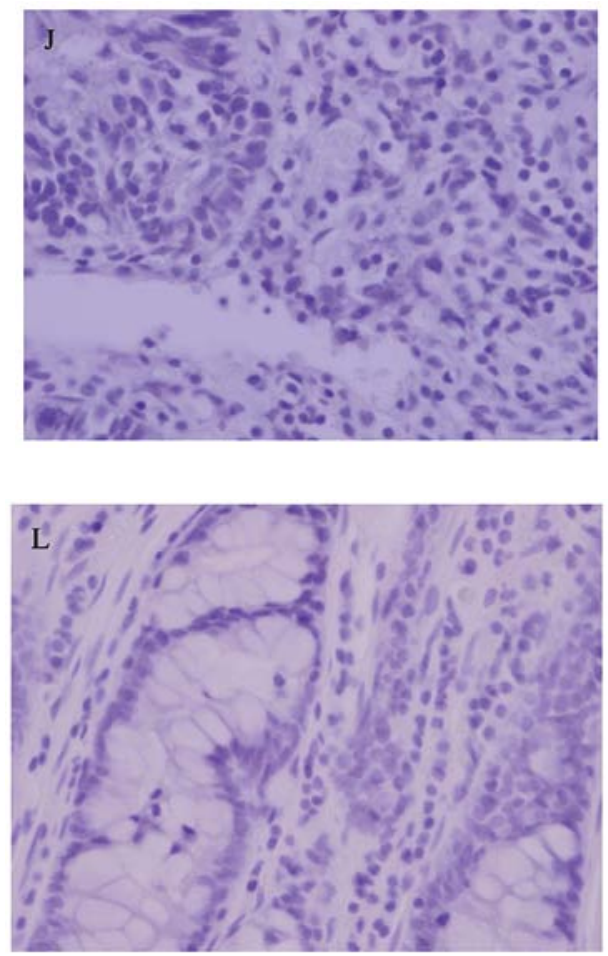
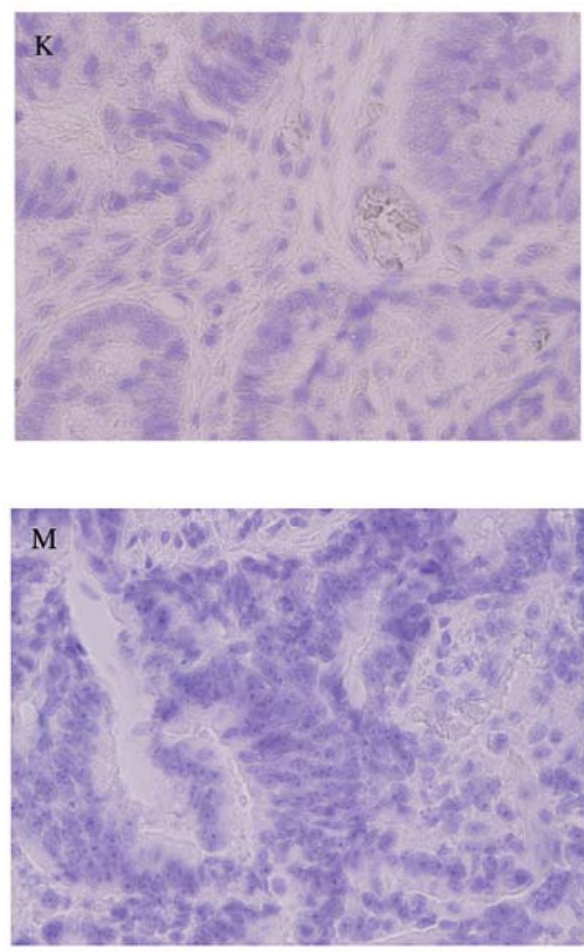

Figure 5. Continued. Immunohistochemical staining for EGFR in resected tumors from 13 colorectal cancer patients (cases J-M).

Table II. The profile of 13 patients with colorectal cancer who underwent surgical resection

\begin{tabular}{clllcccc}
\hline No. & Gender & Location & Stage & EGFR score & KRAS & BRAF & ADCC (\%) \\
\hline 1 & Female & Colon & IIIB & $3+$ & Wild & Wild & 53 \\
2 & Female & Colon & IIIB & $3+$ & Wild & Mutant & 51 \\
3 & Male & Colon & IIIB & $3+$ & Mutant (codon12) & Wild & 50 \\
4 & Female & Rectum & IIIB & $2+$ & Mutant (codon12) & Wild & 79 \\
5 & Male & Colon & IIIB & $2+$ & Mutant (codon13) & Wild & 54 \\
6 & Male & Rectum & II & $2+$ & Wild & Wild & 28 \\
7 & Male & Colon & II & $1+$ & Wild & Wild & 59 \\
8 & Male & Colon & II & $1+$ & Wild & Wild & 30 \\
9 & Female & Rectum & IIIB & $1+$ & Wild & Wild & 20 \\
10 & Female & Colon & II & 0 & Wild & Wild & 26 \\
11 & Male & Colon & II & 0 & Mutant (codon12) & Wild & 9 \\
12 & Male & Rectum & IIIC & 0 & Wild & Wild & 7 \\
13 & Male & Rectum & II & 0 & Wild & Wild & 6 \\
\hline
\end{tabular}

mediated ADCC activity and the cell surface expression level of the EGFR and to determine whether the expression level of the EGFR is a marker for the cetuximab-mediated ADCC activity in vitro using human $\mathrm{CRC}$ cell lines and ex vivo using resected specimens and PBMCs from CRC patients.

The present study revealed novel and important findings relevant to cetuximab-mediated ADCC activity in CRC as follows. First, cetuximab-mediated ADCC activity was correlated with the cell surface expression level of EGFR, but not with the mutational statuses of KRAS and BRAF in CRC cell lines. Second, cetuximab-mediated ADCC activity was also detected in tumor cells from resected specimens and PBMCs isolated from the same patients. Third, cetuximab-mediated ADCC activity was also correlated with the cell surface expression level of EGFR, but not with the mutational statuses of $K R A S$ and $B R A F$ in the resected CRC specimens.

The existence of an acquired EGFR ectodomain mutation (S492R) that prevents cetuximab binding was reported in a previous study (15). Although the incidence is considered to be rare, we made a biotinylated cetuximab to evaluate the cell surface expression level of EGFR in CRC more precisely (11). Biotinylated cetuximab is able to recognize the cell surface 


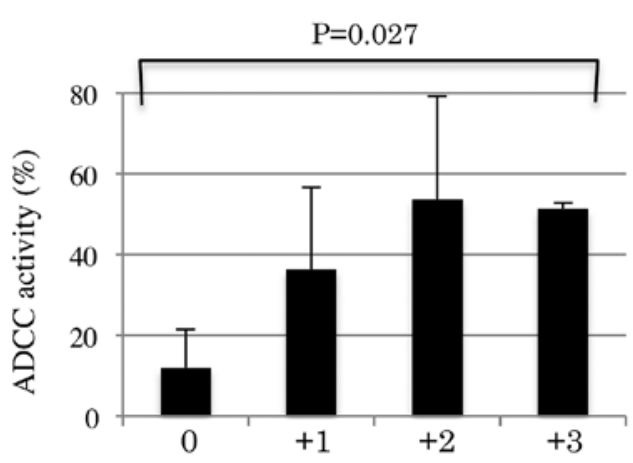

Score of cell surface expression of EGF-R

Figure 6. Cetuximab-mediated ADCC activity of tumor cells from colorectal cancer patients. One-factor ANOVA revealed that high ADCC activities were significantly observed in resected specimens with higher expression levels of cell surface EGFR than in those with lower expression levels $(\mathrm{P}=0.027)$.

EGFR, which is a ligand of cetuximab, even when some mutations in EGFR are present. As a result, the expression levels detected by other commercial anti-human EGFR antibodies were strongly correlated with those detected using biotinylated cetuximab among the CRC cell lines. Therefore, acquired EGFR ectodomain mutations were thought to have a minimal effect on the results of the present study.

The average steady state plasma concentration of cetuximab in cancer patients has been reported to be within the range of 56-100 $\mu \mathrm{g} / \mathrm{ml}$ under the current clinical dose regimen (16). In the present study, a high ADCC activity was detected ex vivo using resected CRC specimens and the patient PBMCs at a cetuximab concentration of $100 \mu \mathrm{g} / \mathrm{ml}$. Therefore, the efficacy of cetuximab-mediated ADCC should be obtained in the current clinical usage.

A previous study revealed that low expression of EGFR might be sufficient for the maximum ADCC activity mediated by cetuximab in lung cancer cell lines (17), whereas another study revealed that a positive correlation between cetuximabmediated ADCC activity and the cell surface expression level of EGFR was observed in human lung cancer, human leukemia and human embryonic kidney cell lines (18). However, the association with ADCC activity mediated by cetuximab and EGFR expression or the KRAS mutational status in CRC has been unclear. In the present study, cetuximab-mediated ADCC activity was strongly and significantly correlated with the cell surface expression level of EGFR, but not with the KRAS/BRAF mutational status, in CRC. In particular, the results of ex vivo experiments using clinical materials from CRC patients could undoubtedly explain one of the mechanisms of action of cetuximab in CRC. Although CRC patients with KRAS mutations are widely known to be able to obtain little benefit from cetuximab therapy, those patients with high levels of cell surface EGFR expression may obtain some benefit from cetuximab treatment. The power balance between the EGFR signaling status and the expression of EGFR on the cell surface, which can induce ADCC, may be important for the efficacy of cetuximab. However, the ADCC activity mediated by cetuximab could not overcome the power of EGFR signaling accelerated by the presence of a KRAS/ $B R A F$ mutation.

\section{References}

1. Kopetz S, Chang GJ, Overman MJ, et al: Improved survival in metastatic colorectal cancer is associated with adoption of hepatic resection and improved chemotherapy. J Clin Oncol 27: 3677-3683, 2009.

2. Marechal R, De Schutter J, Nagy N, et al: Putative contribution of CD56 positive cells in cetuximab treatment efficacy in first-line metastatic colorectal cancer patients. BMC Cancer 10: 340-350, 2010.

3. Tejpar S, Celik I, Schlichting M, Sartorius U, Bokemeyer C and Van Cutsem E: Association of KRAS G13D tumor mutations with outcome in patients with metastatic colorectal cancer treated with first-line chemotherapy with or without cetuximab. J Clin Oncol 30: 3570-3577, 2012.

4. Correale P, Marra M, Remondo C, et al: Cytotoxic drugs up-regulate epidermal growth factor receptor (EGFR) expression in colon cancer cells and enhance their susceptibility to EGFR-targeted antibody-dependent cell-mediated-cytotoxicity (ADCC). Eur J Cancer 46: 1703-1711, 2010.

5. Xu H, Yu Y, Marciniak D, Rishi AK, Sarkar FH, Kucuk O and Majumdar AP: Epidermal growth factor receptor (EGFR)related protein inhibits multiple members of the EGFR family in colon and breast cancer cells. Mol Cancer Ther 4: 435-442, 2005.

6. Yang JL, Qu XJ, Russell PJ and Goldstein D: Regulation of epidermal growth factor receptor in human colon cancer cell lines by interferon $\alpha$. Gut 53: 123-129, 2004.

7. Buck E, Eyzaguirre A, Barr S, et al: Loss of homotypic cell adhesion by epithelial-mesenchymal transition or mutation limits sensitivity to epidermal growth factor receptor inhibition. Mol Cancer Ther 6: 532-541, 2007.

8. Ikehara N, Semba S, Sakashita M, Aoyama N, Kasuga M and Yokozaki H: BRAF mutation associated with dysregulation of apoptosis in human colorectal neoplasms. Int J Cancer 115: 943-950, 2005.

9. Koizumi F, Kanzawa F, Ueda Y, et al: Synergistic interaction between the EGFR tyrosine kinase inhibitor gefitinib ('Iressa') and the DNA topoisomerase I inhibitor CPT-11 (irinotecan) in human colorectal cancer cells. Int J Cancer 108: 464-472, 2004.

10. Balin-Gauthier D, Delord JP, Rochaix P, et al: In vivo and in vitro antitumor activity of oxaliplatin in combination with cetuximab in human colorectal tumor cell lines expressing different level of EGFR. Cancer Chemother Pharmacol 57: 709-718, 2006.

11. Shigeta K, Hayashida T, Hoshino Y, et al: Expression of epidermal growth factor receptor detected by cetuximab indicates its efficacy to inhibit in vitro and in vivo proliferation of colorectal cancer cells. PLoS One 8: e66302, 2013.

12. Wolff A, Hammond M, Schwartz J, et al: American Society of Clinical Oncology/College of American Pathologists guideline recommendations for human epidermal growth factor receptor 2 testing in breast cancer. J Clin Oncol 25: 118-145, 2007.

13. De Roock W, Piessevaux H, De Schutter J, et al: KRAS wild-type state predicts survival and is associated to early radiological response in metastatic colorectal cancer treated with cetuximab. Ann Oncol 19: 508-515, 2008.

14. Steplewski Z, Sun LK, Shearman CW, Ghrayeb J, Daddona P and Koprowski H: Biological activity of human-mouse IgG1, IgG2, IgG3, and IgG4 chimeric monoclonal antibodies with antitumor specificity. Proc Natl Acad Sci USA 85: 4852-4856, 1988.

15. Montagut C, Dalmases A, Bellosillo B, et al: Identification of a mutation in the extracellular domain of the Epidermal Growth Factor Receptor conferring cetuximab resistance in colorectal cancer. Nat Med 18: 221-223, 2012.

16. Luo FR, Yang Z, Dong H, et al: Correlation of pharmacokinetics with the antitumor activity of Cetuximab in nude mice bearing the GEO human colon carcinoma xenograft. Cancer Chemother Pharmacol 56: 455-464, 2005.

17. Kurai J, Chikumi H, Hashimoto K, et al: Antibody-dependent cellular cytotoxicity mediated by cetuximab against lung cancer cell lines. Clin Cancer Res 13: 1552-1561, 2007.

18. Kimura H, Sakai K, Arao T, Shimoyama T, Tamura T and Nishio K: Antibody-dependent cellular cytotoxicity of cetuximab against tumor cells with wild-type or mutant epidermal growth factor receptor. Cancer Sci 98: 1275-1280, 2007. 